Short communication

\title{
Is prenatal maternal distress context-dependent? Comparing United States and the Netherlands
}

\author{
Maria A. Gartstein ${ }^{\mathrm{a}, *}$, Nora L. Erickson ${ }^{\mathrm{b}}$, Kelly H.M. Cooijmans ${ }^{\mathrm{c}}$, Gregory R. Hancock ${ }^{\mathrm{d}}$, \\ Maartje A.C. Zijlmans ${ }^{\mathrm{e}}$, Carolina de Weerth ${ }^{\mathrm{f}}$ \\ ${ }^{\text {a }}$ Washington State University, Vancouver, WA, USA \\ ${ }^{\mathrm{b}}$ Hennepin Healthcare Research Institute, Minneapolis, MN, USA \\ ${ }^{\mathrm{c}}$ Radboud University and Radboud University Medical Center, Nijmegen, The Netherlands \\ ${ }^{\mathrm{d}}$ University of Maryland, College Park, MD 20742, USA \\ ${ }^{\mathrm{e}}$ Radboud University, Nijmegen, The Netherlands \\ ${ }^{\mathrm{f}}$ Radboud University Medical Center, Nijmegen, The Netherlands
}

\section{A R T I C L E I N F O}

\section{Keywords:}

Pregnancy

Anxiety

Depression

Cross-cultural differences

\begin{abstract}
A B S T R A C T
Background: Maternal anxiety and depression symptoms during pregnancy can compromise a woman's wellbeing and affect offspring development. The present study represents a comparison of maternal late-pregnancy internalizing symptoms (i.e., depression and anxiety) between the United States of America (US) and the Netherlands. We hypothesized that women in the US would report higher levels of anxiety and depression during pregnancy compared to their Dutch counterparts, both on individual symptom indicators and overall latent distress, due to more favorable policies/accessible services relevant to perinatal health in the Netherlands. Methods: Pregnant women were recruited at two comparable sites in the Netherlands $(n=327)$ and the US $(n=228)$. Measures included self-reports of internalizing distress and key covariates (i.e., parity, gestational, and maternal age).

Results: Expectant mothers in the US reported higher depressive and anxiety symptoms compared to their Dutch counterparts. Results were consistent across individual internalizing symptom indicators and the overall latent prenatal distress means computed for US and Dutch samples, with an estimated large effect size for the latter after controlling for covariates.

Limitations: Despite their relatively large sizes, our samples were limited in their representativeness of the two cultures and mechanisms contributing to observed differences were not examined.

Conclusions: Pregnant women in the US reported higher levels of depression and anxiety symptoms than women in the Netherlands. Implications concern perinatal policy and clinical services (e.g., emotional health support provided to mothers).
\end{abstract}

\section{Introduction}

Pregnancy is a significant life event associated with physical, emotional, and psychosocial changes. The hormonal and biological fluctuations that occur during pregnancy are thought to increase vulnerability to psychological distress among expectant mothers (Bennett et al., 2004). Along with normative pregnancy-related hormonal shifts, external stressors and adverse circumstances contribute to perceived distress, with the latter altering maternal physiology (e.g., activating the hypothalamic pituitary adrenal or HPA-axis; Beijers et al., 2014), which in turn impacts the offspring (Kingston et al., 2012; Van den Bergh et al., 2017). Prenatal stress has been defined in terms of physiological markers (e.g., cortisol), exposure to significant events (e.g., violence, natural disasters), and also operationalized to include individual and compound symptoms of anxiety and depression (de Weerth, 2018). The latter is a function of widely reported comorbidity between anxiety and depression (Pollack, 2005; Young et al., 2004), and the fact that when experienced during pregnancy both have adverse effects on offspring functioning (Field et al., 2010). A high correspondence between pre- and postnatal symptomatology was previously reported (e.g., Dietz et al., 2007), thus internalizing difficulties experienced during pregnancy are likely to continue impacting postpartum functioning and the transition to parenthood. At the same time, prenatal symptoms of anxiety and depression have been

\footnotetext{
* Corresponding author at: Department of Psychology, Washington State University, P.O. Box 644820, Pullman, WA 99164-4820, USA.

E-mail address: gartstma@wsu.edu (M.A. Gartstein).
} 
associated with unique effects on the offspring, interpreted as programming of the fetus (e.g., Buss et al., 2010; Quarini et al., 2016).

In the present study, we compared state and trait anxiety, along with depressive symptoms between US and Dutch pregnant women. That is, symptoms of prenatal anxiety and depression were examined (a) individually and (b) jointly via a latent mean difference test capturing the compound emotional impact of these internalizing symptoms, referred to as "distress." The focus on internalizing symptoms/ distress during pregnancy, rather than during the postpartum, is a function of the biological programming effects unique to this developmental period (de Weerth, 2018). Importantly, the cross-cultural comparison provides an opportunity to consider whether comparable levels of symptomatology/distress uniformly accompany prenatal transitions, or can be driven by culturally mediated contextual factors, such as access to care and employment (e.g., paid leave, position awaiting return).

Comparing pregnant women from the US and the Netherlands provides a unique opportunity to examine differences between two Western cultures similar in terms of their political and economic circumstances-both are democracies and rate "very high" on the Human Development Index (HDI; United Nations Development Programme, 2014). Moreover, US and the Netherlands are relatively individualistic in their cultural orientation, wherein members of these societies tend to endorse values associated with prioritizing self-interests over those of one's social group (Hofstede et al., 2010). Our prior work addressing cross-cultural differences in infant behavioral outcomes, demonstrating higher levels of negative emotionality and poorer regulation for US infants compared to the Dutch (Desmarais et al., 2017; Sung et al., 2015), also suggested the need to consider maternal functioning. The present dataset provided an opportunity to do so during pregnancy, a period critical because of biological programming implications.

Despite noted cultural similarities, there is substantial variability in workplace and healthcare policy across the two considered countries, including services supporting pregnant women and those available in the postpartum period. For instance, compared to their Dutch counterparts, US women have higher rates of employment throughout pregnancy and return to work more rapidly postpartum (Laughlin, 2011; Vrijkotte et al., 2009). Pregnant women in the Netherlands are guaranteed 16-week paid maternity leave with their job awaiting them; enjoy family-friendly policies, such as part-time work, in order to care for their young children (Addati et al., 2014); and have universal access to child-care assistance in the first postnatal week, which includes home visits, care for the mother, and help with the infant, as needed. Although links between these policies and prenatal distress have not been empirically evaluated, it is plausible that cross-cultural differences in regulations affecting perinatal women may decrease anxiety and depression during pregnancy for the Dutch, compared to US, women. These effects are likely in part because connections between general work stress and symptoms of depression and anxiety have been widely reported (e.g., Melchior et al., 2007). In addition, access to healthcar$\mathrm{e}$-and mental healthcare for mothers in particular-has been noted as a problem in the US (Witt et al., 2009).

There is a sizable literature addressing adversity and distress during pregnancy, with research most relevant to the present study focusing on comparisons across ethnic groups. When comparisons are made within the same country, pregnant women representing minority groups appear to be at greater risk for depression and anxiety (Robinson et al., 2016). Chong et al. (2016) examined anxiety and depression among pregnant women in Singapore from Chinese, Malay, and Indian backgrounds, and found Indian women most likely to meet clinical cutoffs for state anxiety. Considering postpartum depressive symptoms among Vietnamese, Turkish, and Filipino women in Australia, Small et al. (2003) noted higher rates of depression for Turkish women and differences in contributing factors (e.g., Filipino mothers did not mention family issues).
International cross-cultural investigations of psychological distress during pregnancy are most pertinent for the present study. According to Thorpe et al. (1992), Greek women scored significantly higher on measures of prenatal depression and anxiety compared to British pregnant women. These differences were linked to family-related adversity and the transition from a more traditional multi-generational family system, to a modern nuclear-family structure in Greece. In a more recent study, prenatal anxiety and depression symptoms were compared across several European samples and the US (Gorman et al., 2004). Prevalence rates varied from $0 \%$ in Vienna, Austria to $23.3 \%$ in Bordeaux, France with a number of significant differences in "caseness" (e.g., meeting criteria for Generalized Anxiety or Major Depressive Disorder). Swiss and Austrian women exceeded the cut-off criteria less frequently and French women more often, with significantly higher rates of Generalized Anxiety and Major Depressive Disorders observed in Ireland relative to Switzerland. Authors provided several explanations for these differences, including variability in support following delivery. Although informative, this study is limited by small sample sizes ( $n$ s range 20-64; mean $n=32$ ).

The purpose of the present study was to contribute to the literature on international cross-cultural differences in prenatal distress, comparing symptoms of anxiety and depression for US and Dutch women in late pregnancy. Although fetal programming effects earlier in pregnancy have been reported (e.g., Khashan et al., 2008, 2011), the third trimester is a period of rapid development for the brain and HPA-axis, particularly critical to offspring physiology and behavior (Ping et al., 2015; Van den Bergh et al., 2017; Yehuda et al., 2005; Zijlmans et al., 2015). This is also a time when women with limited support and access to care likely experience additional concerns regarding their postpartum circumstances (e.g., return to work), and increases in internalizing symptoms were previously reported during this period (Lee et al., 2007). Existing prenatal distress literature suggests sensitivity to contextual supports (Gorman et al., 2004; Thorpe et al., 1992). Moreover, issues related to mental health care access in the US (Witt et al., 2009) as well as links between work-related stress and depressive/anxiety symptoms (Melchior et al., 2007) have been noted. Thus, we hypothesized that women in the US would report greater anxiety and depression during pregnancy compared to the Dutch on individual symptom indicators and in terms of overall latent distress reflecting combined symptom burden.

\section{Method}

\subsection{Participants}

US: Expectant mothers in their third trimester of pregnancy (i.e., 27-40 weeks gestation) were recruited through a combination of community flyers, advertisements through social media, birthing classes, and hospitals in the Pacific Northwest. This project was approved by the Institutional Review Board at Washington State University as a prospective investigation of links between maternal wellbeing during pregnancy and infant temperament, with informed consent obtained prior to participation. The final sample $(n=228)$ provided all relevant instruments, with exclusions due to medical complications: prenatal hypertension $(n=6)$ and gestational diabetes $(n=4)$, and multiple births $(n=3)$.

Netherlands: The Dutch sample includes data from two studies in comparable groups of pregnant mothers. Inclusion criteria for both studies were an uncomplicated, singleton pregnancy, clear understanding of Dutch language, no drug use, and no current physical health problems. The first group of pregnant women participated in a longitudinal study (BIBO) following healthy mothers and children from late pregnancy (see Beijers et al., 2010 for more details). Of the 220 mothers enrolled, 20 women who began the study after giving birth did not contribute data to the present investigation. This resulted in a final sample of 200 mothers with complete prenatal data. Mothers in the 
second study were part of a randomized controlled trial (SKIPPY) to examine the effectiveness of a skin-to-skin contact intervention in mothers and their full-term infants (see Cooijmans et al., 2017 for more details). This study enrolled 127 mother-infant dyads, with data used in the current investigations collected prior to randomization. Participants were recruited with the cooperation of midwife clinics by handing out flyers in the cities of Nijmegen, Arnhem and surrounding areas (e.g., prenatal exercise and yoga classes, baby fairs). The Ethics Committee of the Faculty of Social Sciences from the Radboud University in Nijmegen approved both original studies, and written informed consent was obtained prior to participation. There were no statistically significant differences on any of the considered symptom indicators or covariates (maternal age, weeks of gestation, and parity) across these two datasets, which were thus combined to form the Dutch sample $(n=327){ }^{1}$

\subsection{Measures}

All of the instruments included in this study have been widely used with US and Dutch samples of pregnant women (e.g., Davis et al., 2007; Huizink et al., 2004; Rode and Kiel, 2016; van den Heuvel, van Assen, Glover, Claes, and Van den Bergh, 2018), and in perinatal research including cross-cultural comparisons (e.g., Chong et al., 2016).

The Edinburgh Postnatal Depression Scale (EPDS; Cox et al., 1987) is a 10-item self-report measure of depression in the perinatal period. The EPDS has been utilized in clinical and research contexts (Cox et al., 1987) and validated for use during pregnancy (Bennett et al., 2004; Murray and Cox, 1990). The EPDS had good internal consistency in the present US ( $\alpha=0.89$ ) and Dutch samples ( $\alpha=0.81$ ).

The State Trait Anxiety Inventory (STAI; Spielberger et al., 1989) represents a self-report measure of anxiety. This instrument consists of two forms (one for each anxiety construct), which include 20 statements each. The questionnaire asks the rater either to describe how she feels at a certain moment in time (state-anxiety) or how she generally feels (trait-anxiety). State anxiety is conceptualized as a time-limited emotional state that includes feelings of tension and apprehensiveness and trait-anxiety is viewed as more stable, with both scores examined in studies addressing prenatal stress (e.g., Chung et al., 2016). Previous research demonstrated good internal consistency for STAI state and trait indicators (Cronbach's $\alpha=0.91$ for each scale; Spielberger et al., 1989). In the present US sample, the STAI state anxiety index had excellent internal consistency ( $\alpha=0.93)$, as did as the trait anxiety indicator $(\alpha=0.97)$. In the Dutch sample, reliability was also good for both state $(\alpha=0.85)$ and trait $(\alpha=0.83)$ scales.

\subsection{Analytic strategy}

Analyses were aimed at discerning differences between pregnancy experiences of anxiety and depression for US and Dutch women. They involved comparisons of individual internalizing symptom indicators as well as quantification of differences in the overall levels of distress. Following Fan and Hancock (2012), the structured means modeling analogs to independent groups $t$-tests were conducted, followed by the inclusion of covariates to control for maternal age, weeks of gestation, and parity. These analyses do not make any assumptions of homogeneity of (residual) variance across groups; further, we invoked a Satorra-Bentler rescaling correlation to accommodate any potential nonnormality. Covariates were included in analyses to ensure that observed differences were not a function of these factors related to prenatal depression, anxiety, and stress, yet not central to the hypotheses examined in this study. Links between prenatal distress and duration of pregnancy, as well as relations with maternal age and parity, have been reported (Biaggi et al., 2016; Gillespie et al., 2018; Lee et al., 2007;

\footnotetext{
${ }^{1}$ Results of analyses comparing two Dutch samples available from the 1 st author upon request.
}

Lorén-Guerrero et al., 2018; Marchesi et al., 2009). For example, a recent review noted that younger and older mothers were shown to be atrisk of internalizing symptoms, and that related distress was often elevated later in pregnancy, possibly because women experience increased symptoms as the transition to parenthood approaches (Biaggi et al., 2016).

Finally, latent mean differences were examined, providing comparisons of the overall latent distress resulting from internalizing symptoms assessed in this study, also ensuring covariates were not responsible for observed differences (Hancock, 2001, 2004). Briefly, measured variable techniques such as analysis of (co)variance may underestimate the magnitude of group differences, and attenuate power to detect such differences, due to measurement error in the outcome variable. Latent means models, an extension of structural equation models, incorporate a factor measurement model along with a mean structure, thus parsing measurement error from the assessment of any group mean differences. The resulting latent mean difference is thus a direct estimate of the degree of separation along the latent (i.e., errorfree) continuum of interest, in this case distress, that would have to exist in order to precipitate the group differences observed on the individual indicators of the factor/latent variable. This is a purer estimate of the actual separation of groups on the outcome of interest; further, this analytical framework allows for the inclusion of covariates (measured or latent), the accommodation of missing data, as well as robust corrections for potential non-normality. For more information about this versatile method, the reader is referred to Hancock (2004) or Thompson and Green (2013). ${ }^{2}$

\section{Results}

Descriptive statistics for covariates and dependent variables were computed first for US and Dutch samples separately (Table 1). Robust structured means models were used for independent groups mean comparisons with prenatal depressive symptoms, state and trait anxiety as dependent variables. All three comparisons were statistically significant and US women reported greater symptomatology with medium effect sizes for the observed differences. For EPDS $(z=6.63, p<.001)$,

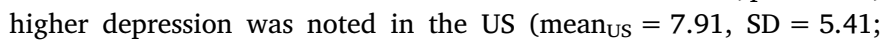
mean $_{\mathrm{NL}}=5.08, \quad \mathrm{SD}=4.19 ; \mathrm{ES}=0.60$ ). For STAI state anxiety $(z=5.92, p<.001)$, US women's ratings were significantly higher $\left(\right.$ mean $_{\mathrm{US}}=36.94, \quad \mathrm{SD}=11.69 ; \quad \operatorname{mean}_{\mathrm{NL}}=31.41, \quad \mathrm{SD}=8.60$; $\mathrm{ES}=0.56)$, with a similar pattern of results $(z=5.49, p<.001)$ for the trait indicator $\left(\operatorname{mean}_{\mathrm{US}}=37.34, \quad \mathrm{SD}=11.52 ; \quad \operatorname{mean}_{\mathrm{NL}}=32.40\right.$, $\mathrm{SD}=8.26$; $\mathrm{ES}=0.52$ ). ANCOVAs provided further evidence of significant cross-cultural differences (Table 2), also indicating medium effect sizes.

Latent mean differences were also considered, with the latent prenatal distress encompassing EPDS and STAI state and trait indicators, modeled with Mplus 8 (Muthén and Muthén, 2017) using Full Information Maximum Likelihood estimation to accommodate missing data and robust corrections to account for non-normality. We initially examined a basic/unconditional latent mean model, with loadings and intercepts constrained to be invariant across groups, and subsequently included maternal age, weeks of gestation, and parity as covariates influencing latent prenatal distress. The basic prenatal distress latent mean model provided a good data-model fit $\left(\chi^{2}=6.20\right.$, NS; RMSEA $=0.00$; SRMR $=0.02$ ), supporting measurement invariance and demonstrating a statistically significant difference between the US and the Netherlands on latent prenatal distress. The final model including covariates also demonstrated good fit $\left(\chi^{2}=13.16\right.$, NS; RMSEA $=0.00$; SRMR $=0.02$ ), again indicating that measurement invariance constraints were appropriate for the data. All three indicators were associated with strong and statistically significant loadings

\footnotetext{
${ }^{2}$ Mplus code used in analyses will be made available to upon request.
} 
Table 1

Descriptive statistics for the US and Dutch samples.

\begin{tabular}{|c|c|c|c|c|}
\hline $\begin{array}{l}\text { US }(N=228) \\
\text { Variable }\end{array}$ & Mean & SD & Range & Percentage \\
\hline Prenatal Depression/EPDS & 7.91 & 5.41 & $0-28$ & \\
\hline Prenatal State Anxiety/STAI & 34.94 & 11.69 & $20-76$ & \\
\hline Prenatal Trait Anxiety/STAI & 37.34 & 11.52 & $20-71$ & \\
\hline Maternal Age (Years) & 27.66 & 4.93 & $18-40$ & \\
\hline Weeks of Gestation & 32.16 & 4.05 & $27-40$ & \\
\hline \multicolumn{5}{|l|}{ Parity } \\
\hline Primiparous & & & & $59.2 \%$ \\
\hline Multiparous & & & & $40.8 \%$ \\
\hline \multicolumn{5}{|l|}{ Dutch $(N=327)$} \\
\hline Variable & Mean & SD & Range & Percentage \\
\hline Prenatal Depression (EPDS) & 5.08 & 4.19 & $0-21$ & \\
\hline Prenatal State Anxiety (STAI) & 31.41 & 8.60 & $20-64$ & \\
\hline Prenatal Trait Anxiety (STAI) & 32.40 & 8.26 & $21-68$ & \\
\hline Maternal Age & 32.21 & 3.74 & $21-43$ & \\
\hline Weeks of Gestation & 38.42 & 2.22 & $32-42$ & \\
\hline \multicolumn{5}{|l|}{ Parity } \\
\hline Primiparous & & & & $44.2 \%$ \\
\hline Multiparous & & & & $55.8 \%$ \\
\hline
\end{tabular}

greater heterogeneity in the levels of overall distress resulting from internalizing symptoms experienced by this group.

\section{Discussion}

Cross-cultural differences in prenatal depressive symptoms and state and trait anxiety were observed, with expectant mothers in the US reporting higher levels compared to their Dutch counterparts. This difference was evident across individual symptom indicators and the overall latent prenatal distress means, with the latter demonstrating a large effect size for compound internalizing problems, controlling for covariates. This pattern of results is consistent with prior studies demonstrating significant cross-cultural differences in prenatal distress (Gorman et al., 2004; Thorpe et al., 1992), indicating sensitivity to contextual factors. As noted, rates of prenatal anxiety and depression varied across several European samples (Gorman et al., 2004), and although women from the Netherlands were not included, explanations for observed differences focused on variability in perinatal support are relevant to interpreting results of the present study. Our findings indicate that expectant mothers in the US would likely benefit from additional services, including but not limited to support from health care providers. Paraprofessional services (e.g., access to doulas or birth

Table 2

Covariate beta weights for the US/Netherlands and culture standardized effect size.

\begin{tabular}{|c|c|c|c|c|}
\hline & Maternal age & Gestational age & Parity & Culture \\
\hline Prenatal Depression (EPDS) & $-0.105 * /-0.102 *$ & $0.066 / 0.046$ & $0.065 \# / 0.084 \#$ & $0.64^{* * *}$ \\
\hline Prenatal State Anxiety (STAI) & $-0.089 \# /-0.092^{\#}$ & $0.088^{\#} / 0.066^{\#}$ & $0.070^{*} / 0.096^{*}$ & $0.66^{* * *}$ \\
\hline Prenatal Trait Anxiety (STAI) & $-0.040 /-0.042$ & $-0.002 /-0.002$ & $0.058 \# / 0.081^{\#}$ & $0.49^{*: * * * *}$ \\
\hline
\end{tabular}

*** $p<.001$.

${ }^{*} p<.05$.

\# $p<.10$.

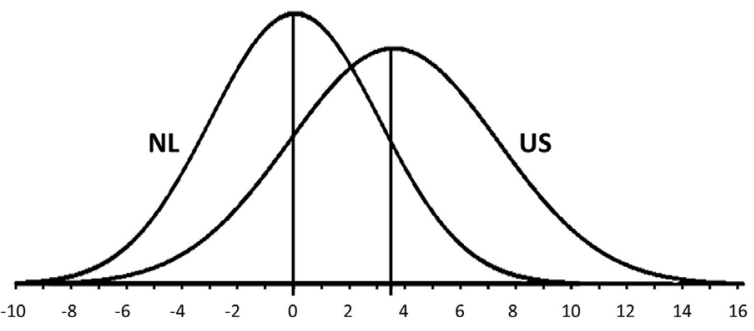

LATENT PRENATAL DISTRESS CONTINUUM (scaled using EPDS units)

Fig. 1. US and Dutch expectant mothers latent prenatal distress.

ranging from 0.77 to 0.96 , but none of the covariates made a statistically significant contribution to latent prenatal distress (all $p$ 's $>.10$ ). A statistically significant difference $(p<.001)$ for US and Dutch expectant mothers was observed on latent prenatal distress. Pregnant women in the US self-reported higher overall distress relative to Dutch expectant mothers (Fig. 1). Based on the latent mean estimates and variances after controlling for the covariates (and using the EPDS metric to assign scale to the factor), the US was estimated to be almost a standard deviation higher than NL on the latent distress continuum (standardized latent effect size $=0.85$ ), after accounting for covariates (latent $\operatorname{mean}_{\mathrm{NL}}=0, s^{2}=10.67$; latent $\left.\operatorname{mean}_{\mathrm{US}}=3.53, s^{2}=26.76\right)^{3}$ Greater latent variance of the US sample also suggests considerably

\footnotetext{
${ }^{3}$ Interactions involving culture and covariates were examined via a series of multiple regression analyses, and significant interaction effects were not observed.
}

coaches) could be an important component to ensuring perinatal mental and physical health.

Additional research is required to identify specific causes, as mechanisms were not addressed in the current investigation; however, relevant workplace and healthcare policy differences between the US and the Netherlands may contribute. That is, expectant mothers in the US are confronted with concerns related to access to services (i.e., both health and childcare) and limited employment accommodations, such as lack of universal paid leave. In comparison, Dutch women enjoy a variety of family-friendly policies, such as no-cost doula services, that can be expected to translate into differential pregnancy experiences and associated levels of internalizing symptoms. Moreover, universal healthcare available in the Netherlands, including mental/behavioral health (van de Ven and Schut, 2008) likely contributed to the observed pattern of results. Despite their apparent relevance, such factors have not been examined as predictors of prenatal distress in the existing literature. However, mental health care access is a widely documented concern in the US, especially for mothers, with only $34.8 \%$ of a population-based sample who reported depression receiving adequate treatment (Witt et al., 2009). As noted, work stress generally contributes to depression and anxiety symptoms (Melchior et al., 2007), and women are likely to find negotiating pregnancy/child-birth related workplace absences stressful. Of course, other relevant variables, including lifestyle factors (e.g., dietary patterns, health behaviors during pregnancy) and partner support may differ among these two cultures and should be considered.

These results await replication and extension yet deserve attention due to implications for mothers, children, and families. Postpartum distress has received greater attention in the literature, yet prenatal depression and anxiety represent significant risk factors for postnatal symptoms (Norhayati et al., 2015). Not only is prenatal distress 
associated with compromised maternal well-being, it can be responsible for fetal programming and altered offspring developmental outcomes (e.g., Van den Bergh, et al., 2017). Although the present study does not address underlying biological mechanisms, it provides preliminary evidence that such programming effects may differ between the US and the Netherlands-two developed countries similar in terms of the socioeconomic context and cultural orientation. Increased offspring negative emotionality represents a primary behavioral consequence of fetal programming due to prenatal distress (Beijers et al., 2014), as Davis et al. (2007), for example, reported increased infant negative emotionality as a function of exposure to elevated maternal anxiety and depression during pregnancy. Prior work has provided evidence of cross-cultural differences in negative emotionality between US and Dutch infants based on reports of mothers and fathers from independent samples (Desmarais et al., 2017; Sung et al., 2015), with higher overall negative emotionality, fear, frustration, and sadness for US infants. Such differences are typically attributed to culturally-influenced caregiving; yet, prenatal exposure to depression and anxiety, and associated programming effects, provide another plausible explanation. Identification of factors contributing to this temperament phenotype that confers risk for emotional and behavioral problems (e.g., Eisenberg et al., 2005; Gartstein et al., 2012) is imperative, and exposure to prenatal distress should be considered, as this pathway represents preventable risk.

This study has a number of limitations. First, despite relatively large samples, representativeness of the two cultures was limited and future research should obtain data from multiple sites within each culture. Second, we were not able to control for socio-economic status; however, our prior work drawing samples from the same communities produced groups comparable in terms of occupation-based socio-economic indicators and education (Gartstein and Putnam, 2018; Sung et al., 2015). Lifestyle variables, dietary patterns, and partner support, may differ cross-culturally contributing to well-being during pregnancy, and should be considered. Importantly, future research should directly address policy/accessibility factors implicated by the present study. In addition, only maternal reports of depression- and anxiety-related symptoms in the third trimester were obtained. Future studies should include bio-markers of stress reactivity and partner reports of prenatal psychological well-being across pregnancy. Although internalizing symptoms addressed in this study often occur in response to stressful circumstances, the latter were not examined per se, and should be evaluated in the future.

In conclusion, pregnant mothers in the US reported more pregnancy depression and anxiety complaints than expectant women in the Netherlands. Noted differences may at least partly explain previously identified cross-cultural differences in infant temperament. These are also likely relevant for understanding how pregnancy and postpartum policies and services affect maternal psychological wellbeing during pregnancy.

\section{Role of the funding source}

Research conducted in the US was made possible by the 2015 Marchionne Summer Research Fellowship from Washington State University (Nora Erickson, PI). For the Dutch sample, majority of participants were involved the BIBO study, funded by the NWO (Netherlands Organisation for Scientific Research) personal Vidi grant -Innovational Research Incentives Scheme- (2005-2010) "In search of the roots of self-regulation: a biobehavioral approach" to Carolina de Weerth, PI. A portion participated in the SKIPPY study, funded by the Behavioural Science Institute Graduate School, Radboud University Nijmegen (2015-2019) PhD grant: "Skin-to-skin contact to improve outcomes in mothers and their full-term infants: A randomized controlled trial" (Carolina de Weerth, PI).

\section{CRediT authorship contribution statement}

Maria A. Gartstein: Writing - original draft, Project administration. Nora L. Erickson: Writing - original draft, Data curation. Kelly H.M. Cooijmans: Writing - original draft, Data curation. Gregory R. Hancock: Writing - original draft, Formal analysis. Maartje A.C. Zijlmans: Writing - original draft, Data curation. Carolina de Weerth: Writing - original draft, Project administration.

\section{Declaration of Competing Interest}

None of the co-authors participating in this research and development of the submitted manuscript have any actual or potential conflicts of interest to disclose, including any financial, personal or other relationships.

\section{Acknowledgements}

We gratefully acknowledge the contribution of participating women and the help of multiple research assistants in the United States (US) and the Netherlands.

\section{Supplementary materials}

Supplementary material associated with this article can be found, in the online version, at doi:10.1016/j.jad.2019.09.048.

\section{References}

Addati, L., Cassirer, N., Gilchrist, K., 2014. Maternity and Paternity at Work: Law and Practice Across the World. International Labor Office, Geneva, Austria.

Beijers, R., Buitelaar, J.K., de Weerth, C., 2014. Mechanisms underlying the effects of prenatal psychosocial stress on child outcomes: beyond the HPA axis. Eur. Child Adolesc. Psychiatry 23, 943-956.

Beijers, R., Jansen, J., Riksen-Walraven, M., de Weerth, C., 2010. Maternal prenatal anxiety and stress predict infant illnesses and health complaints. Pediatrics 126 , e401-e409.

Bennett, H.A., Einarson, A., Taddio, A., Koren, G., Einarson, T.R., 2004. Prevalence of depression during pregnancy: systematic review. Obstet.Gynecol. 103, 698-709.

Biaggi, A., Conroy, S., Pawlby, S., Pariante, C.M., 2016. Identifying the women at risk of antenatal anxiety and depression: a systematic review. J. Affect. Disord. 191, 62-77.

Buss, C., Davis, E.P., Muftuler, T., Head, K., Sandman, C.A., 2010. High pregnancy anxiety during mid-gestation is associated with decreased gray matter density in 6-9 year-old children. Psychoneuroendocrinology 35, 141-153.

Chong, S.C., Broekman, B.F.P., Qiu, A., Aris, I.M., Chan, Y.H., Rifkin-Graboi, A., Law, E., Chee, C.Y.I., 2016. Anxiety and depression during pregnancy and temperament in early infancy: findings from a multi-ethnic, Asian, prospective birth cohort study. J. Infant Mental Health 37, 584-598.

Cox, J.L., Holden, J.M., Sagovsky, R., 1987. Detection of postnatal depression: development of the 10-item Edinburgh postnatal depression scale. Br. J. Psychiatry 15, $782-786$.

Cooijmans, H.H.M., Beijers, R., Rovers, A.C., Weerth, C., 2017. Effectiveness of skin-toskin contact versus care-as-usual in mothers and their full-term infants: study protocol for a parallel-group randomized controlled trial. BMC Pediatrics 17, 154. https://doi.org/10.1186/s12887-017-0906-9.

Davis, E.P., Glynn, L.M., Dunkel Schetter, C., Hobel, C., Chicz-DeMet, A., Sandman, C.A., 2007. Prenatal exposure to maternal depression and cortisol influences infant temperament. J. Am.Acad. Child Adolesc. Psychiatry 46, 737-746.

Desmarais, E., Majdandzic, M., Gartstein, M.A., Bridgett, D.J., French, B., 2017. Crosscultural differences in temperament: comparing paternal ratings of US and Dutch infants. Eur. J Dev. Psychol. https://doi.org/10.1080/17405629.2017.1356713.

de Weerth, C., 2018. Prenatal stress and the development of psychopathology: lifestyle behaviors as a fundamental part of the puzzle. Dev. Psychopathol. 30, 1129-1144. https://doi.org/10.1017/S0954579418000494.

Dietz, P.M., Williams, S.B., Callaghan, W.M., Bachman, D.J., Whitlock, E.P., Hornbrook, M.C., 2007. Clinically identified maternal depression before, during, and after pregnancies ending in live births. Am. J. Psychiatry 164, 1515-1520.

Eisenberg, N., Sadovsky, A., Spinrad, T.L., Fabes, R.A., Losoya, S.H., Valiente, C., Reiser, M., Cumberland, A., Shepard, S.A., 2005. The relations of problem behavior status to children's negative emotionality, effortful control, and impulsivity: concurrent relations and prediction of change. Dev. Psychol. 41, 193-211.

Fan, W., Hancock, G.R., 2012. Robust means modeling: an alternative to hypothesis testing of independent means under variance heterogeneity and nonnormality. J. Educ. Behav Stat. 37, 137-156.

Field, T., Diego, M., Hernandez-Reif, M., Figueiredo, B., Deeds, O., Ascencio, A., Schanberg, S., Kuhn, C., 2010. Comorbid depression and anxiety effects on pregnancy and neonatal outcome. Infant Behav. Dev. 33, 23-37. 
Gartstein, M.A., Putnam, S.P., 2018. Temperament, Parents and Culture: Findings from the Joint Effort Toddler Temperament Consortium (JETTC). Routledge, New York, NY.

Gartstein, M.A., Putnam, S.P., Rothbart, M.K., 2012. Etiology of preschool behavior problems: contributions of temperament attributes in early childhood. Infant Ment. Health J. 33, 197-211.

Gillespie, S.L., Mitchell, A.M., Kowalsky, J.M., Christian, L.M., 2018. Maternal parity and perinatal cortisol adaptation: the role of pregnancy specific distress and implications for postpartum mood. Psychoneuroendocrinology 97, 86-93.

Gorman, L.L., O'hara, M.W., Figueiredo, B., Hayes, S., Jacquemain, F., Kammerer, M.H., Klier, C.M., Rosi, S., Seneviratne, G., Sutter-Dallay, A.L., the TCS-PND Group, 2004. Adaptation of the structured clinical interview for DSM-IV disorders for assessing depression in women during pregnancy and post-partum across countries and cultures. Br. J. Psychiatry 184, s17-s23.

Hancock, G.R., 2001. Effect size, power, and sample size determination for structured means modeling and MIMIC approaches to between-groups hypothesis testing of means on a single latent construct. Psychometrika 66, 373-388.

Hancock, G.R., 2004. Experimental, quasi-experimental, and nonexperimental design and analysis with latent variables. In: Kaplan, D. (Ed.), The SAGE Handbook of Quantitative Methodology for the Social Sciences. SAGE Publications, Thousand Oaks, CA, pp. 317-334.

Hofstede, G., Hofstede, G.J., Minkov, M., 2010. Cultures and Organizations: Software of the Mind. McGraw-Hill, New York Rev. third ed.

Huizink, A.C., Mulder, E.J., Robles de Medina, P.G., Visser, G.H., Buitelaar, J.K., 2004. Is pregnancy anxiety a distinctive syndrome? Early Hum. Dev. 79, 81-91.

Khashan, A.S., Abel, K.M., McNamee, R., Pedersen, M.G., Webb, R.T., et al., 2008. Higher risk of offspring schizophrenia following antenatal maternal exposure to severe adverse life events. Arch. Gen. Psychiatry 65, 146-152.

Khashan, A.S., McNamee, R., Henriksen, T.B., Pedersen, M.G., Kenny, L.C., et al., 2011. Risk of affective disorders following prenatal exposure to severe life events: a Danish population-based cohort study. J. Psychiatr. Res. 45, 879-885.

Kingston, D., Tough, S., Whitfield, H., 2012. Prenatal and postpartum maternal psychological distress and infant development: a systematic review. Child Psychiatry Hum. Dev. 43, 683-714.

Lorén-Guerrero, L., Gascón-Catalán, A., Pasierb, D., Romero-Cardiel, M.A., 2018. Assessment of significant psychological distress at the end of pregnancy and associated factors. Arch. Womens Ment Health 21, 313-321.

Laughlin, L., 2011. Maternity leave and employment patterns: 2006-2008. Current Population Report, P70-128. U.S. Census Bureau, Washington, DC.

Lee, A.M., Lam, S.K., Sze Mun Lau, S.M., Shiu Yin Chong, C., Chui, H.W., Yee Tak Fong, D., 2007. Prevalence, course, and risk factors for antenatal anxiety and depression. Obstet. Gynecol. 110, 1002-1012.

Marchesi, C., Bertoni, S., Maggini, C., 2009. Major and minor depression in pregnancy. Obstet. Gynecol. 113, 1292-1298.

Melchior, M., Caspi, A., Milne, B.J., Danese, A., Poulton, R., Moffitt, T.E., 2007. Work stress precipitates depression and anxiety in young, working women and men. Psychol. Med. 37, 1119-1129.

Murray, D., Cox, J.L., 1990. Screening for depression during pregnancy with the Edinburgh Depression Scale (EPDS). J. Reprod. Infant. Psyc. 8 (2), 99-107.

Muthén, L.K., Muthén, B.O., 2017. Mplus 8 [Software]. Available from. http:// statmodel.com.

Norhayati, M.N., Hazlina, N.H., Asrenee, A.R., Emilin, W.M., 2015. Magnitude and risk factors for postpartum symptoms: a literature review. J. Affect. Disord. 175, 34-52.

Quarini, C., Pearson, R.M., Stein, A., Ramchandani, P.G., Lewis, G., Evans, J., 2016. Are female children more vulnerable to the long-term effects of maternal depression during pregnancy? J. Affect. Disord. 189, 329-335.

Ping, Y.E., Laplante, D.P., Elgbeili, G., Hillerer, K.M., Brunet, A., O'Hara, M.W., King, S., 2015. Prenatal maternal stress predicts stress reactivity at 2(1/2) years of age: the iowa flood study. Psychoneuroendocrinology 56, 62-78.

Pollack, M., 2005. Comorbid anxiety and depression. J. Clin. Psychiatry 66, 22-29.

Robinson, A.M., Benzies, K.M., Cairns, S.M., Fung, T., Tough, S.C., 2016. Who is distressed? a comparison of psychosocial stress in pregnancy across seven ethnicities. BMC Pregnancy Childbirth 16, 215-226.

Rode, J.L., Kiel, E.J., 2016. The mediated effects of maternal depression and infant temperament on maternal role. Arch. Womens Ment. Health 19, 133-140.

Small, R., Lumley, J., Yelland, J., 2003. Cross-cultural experiences of maternal depression: associations and contributing factors for Vietnamese, Turkish and Filipino immigrant women in Victoria, Australia. Ethn. Health 8, 189-206.

Spielberger, C.D., Gorsuch, R.L., Lushane, R., Vagg, P.H., Jacobs, G.A., 1989. Manual for the State Trait Anxiety Inventory (form Y). University of South Florida, Tampa, FL.

Sung, J., Beijers, R., Gartstein, M.A., de Weerth, C., Putnam, S., 2015. Exploring temperamental differences in infants from the United Sates of America (US) and the Netherlands. Eur. J. Dev. Psychol. 12, 15-28.

Thompson, M.S., Green, S.B., 2013. Evaluating between-group differences in latent variable means. In: Hancock, G.R., Mueller, R.O. (Eds.), Structural Equation Modeling: A Second Course. Information Age Publishing, Charlotte, NC, pp. 163-218.

Thorpe, K.J., Dragonas, T., Golding, J., 1992. The effects of psychosocial factors on the emotional well-being of women during pregnancy: a cross-cultural study of britain and greece. J. Reprod. Infant Psychol. 10, 91-104.

United Nations Development Programme, 2014. Human Development Report 2014 Sustaining Human Progress: Reducing Vulnerabilities and Building Resilience. United Nations Development Programme, New York.

van de Ven, W.P.M.M., Schut, F.T., 2008. Universal mandatory health insurance in the Netherlands: a model for the United States? Health Aff. 27, 771-781.

Van den Bergh, B.R.H., van den Heuvel, M.I., Lahti, M., Braeken, M., de Rooij, S.R., ... Schwab, M., 2017. Prenatal developmental origins of behavior and mental health: the influence of maternal stress in pregnancy. Neurosci. Biobehav. Rev. http://dx.doi. org/10.1016/j.neubiorev.2017.07.003.

van den Heuvel, M.I., van Assen, M.A.L.M., Glover, V., Claes, S., Van den Bergh, B.R.H., 2018. Associations between maternal psychological distress and salivary cortisol during pregnancy: a mixed-models approach. Psychoneuroendocrinology 96, 52-60.

Vrijkotte, T.G.M., van der Wal, M.F., van Eijsden, M., Bonsel, G.J., 2009. First-trimester working conditions and birthweight: a prospective cohort study. Am. J. Public Health 99, 1409-1416.

Witt, W.P., Keller, A., Gottlieb, C., Litzelman, K., Hampton, J., Maguire, J., Hagen, E.W., 2009. Access to adequate outpatient depression care for mothers in the USA: a nationally representative population-based study. J. Behav. Health Ser. Res. 38, 191-204.

Yehuda, R., Engel, S.M., Brand, S.R., Seckl, J., Marcus, S.M., Berkowitz, G.S., 2005 Transgenerational effects of posttraumatic stress disorder in babies of mothers exposed to the World Trade Center attacks during pregnancy. J. Clin. Endocrinol. Metab. 90, 4115-4118.

Young, E., Abelson, J., Cameron, O., 2004. Effect of comorbid anxiety disorders on the hypothalamic-pituitary-adrenal axis response to a social stressor in major depression. Biol. Psychiatry 56, 113-120.

Zijlmans, M.A.C., Riksen-Walraven, J.M., de Weerth, C., 2015. Associations between maternal prenatal cortisol concentrations and child outcomes: a systematic review. Neurosci Biobehav. Rev 53, 24-30. 